\title{
The Solutions Come from Science and Knowledge
}

\author{
Johannes Czernin and Caius Radu \\ David Geffen School of Medicine at UCLA, Los Angeles, California
}

$\mathbf{J}$ odi Doering, a nurse from South Dakota, tweeted that many patients dying from COVID-19 infections did not believe that the virus was real. Bill Gates expressed disbelief about vaccination conspiracy theories including that he was behind the coronavirus pandemic to microchip and control the population. A survey of 26,000 people in 25 countries conducted by YouGov-Cambridge Globalism Project in collaboration with The Guardian revealed that $40 \%$ of U.S. Americans believe that the COVID-19 fatality rate was exaggerated. Thirty percent of U.S. respondents expressed safety concern about vaccines in general.

The argument that mandated mask wearing and social distancing are interfering with personal freedom is an international phenomenon. A recent mass demonstration in Berlin, Germany, had to be dispersed by use of water cannons. Demonstrators, in their absurd disregard for safety of others, refused to wear masks or adhere to social distancing. Countries, states, and courts have a difficult time enforcing protective measures, and mandated COVID-19 vaccinations would likely result in mass demonstrations.

The philosopher Karl Popper proposed the paradox of tolerance in The Open Society and Its Enemies (published in 1945): "Unlimited tolerance must lead to the disappearance of tolerance. If we extend unlimited tolerance even to those who are intolerant, if we are not prepared to defend a tolerant society against the onslaught of the intolerant, then the tolerant will be destroyed, and tolerance with them. [...] We should therefore claim, in the name of tolerance, the right not to tolerate the intolerant" (1).

More than 20 million U.S. residents have been infected with COVID-19, and more than 350,000 people have died in the United States. Astonishingly, close to $40 \%$ of all COVID-19 cases in the United States occurred in November and December, reflecting an exponential, largely uncontrolled nationwide spread of the virus, which shows no signs of abating. Much of this was avoidable if common decency and respect and regard for others had governed behavior.

It is, of course, not surprising that the solution to the COVID-19 pandemic will ultimately come from groundbreaking scientific achievements that have occurred at a truly unprecedented rate, rivaling the exponential growth of the virus. A study from the Massachusetts Institute of Technology has tracked 60,000-80,000 peer-reviewed publications on COVID-19 since the beginning of 2020, an enormous number reflecting a mobilization of science on a scale never before seen. Two vaccines, one from Pfizer/BioNTech and the other from Moderna, have already successfully completed phase 3 clinical trials and are on track to receive emergency-use authorization from the Food and Drug Administration in December, followed by an immediate rollout of tens of millions of doses to highest-priority groups. These 2 vaccines have been reported to be remarkably efficacious (only data for the Pfizer vaccine have been fully published (2)), but they also use an entirely novel approach based on messenger RNA, which has the promise

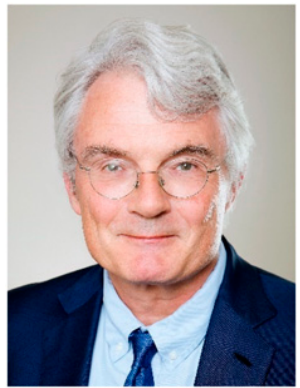

Johannes Czernin to revolutionize vaccine development and transform our ability to rapidly and efficiently respond to future emerging pandemics.

Regarding therapeutics, after initial missteps mainly related to the hydroxychloroquine saga, which bizarrely continues to this day in the halls of the U.S. Senate, and the overly optimistic promotion of remdesivir, 2 types of monoclonal antibody cocktails developed by Eli Lily and Regeneron have received emergencyuse authorization and are being deployed to hospitals around the United States. These scientific developments are just the beginning, with hundreds of vaccine and therapy candidates in various stages of development. As Larry Brilliant, an expert on pandemics who helped eradicate smallpox, noted in WIRED magazine, "we now live through the best of times [as far as science's beneficial impact on society is concerned], and the worst of times [from an epidemiological perspective with horrifying increases in virus spread and hospitalizations]" (3).

Since humans are responsible for both sides of this Dickensian paradox, it is therefore in our power to choose science and reason over misinformation and irrationality, and, in the process, dramatically limit the terrible impact of this disease. It is certainly not too late to make the right choices.

\section{REFERENCES}

1. Popper K. The Open Society and Its Enemies. Routledge; 1945:265.

2. Polack FP, Thomas SJ, Kitchin N, et al. Safety and efficacy of the BNT162b2 mRNA Covid-19 vaccine. N Engl J Med. 2020;383:2603-2615.

3. Levy S. Larry Brilliant says we'll beat Covid-after we go through hell. WIRED website. https://www.wired.com/story/larry-brilliant-says-we-will-beat-covid-after-wego-through-hell/. November 18, 2020. Accessed December 31, 2020. 